\title{
A NEW METHOD FOR THE EXAMINATION OF COFFEE.
}

By F. M. Rimuington, F.C.S., F.I.C.

I THINK it will be generally admitted that the methods in use for estimating the degree of adulteration in coffee are far from satisfactory as regards definiteness and certainty, and that something more approaching to chemical accuracy is very desirable. Little has been done in this direction since the days of the Lancet Sanitary Commission.

It may not be generally known to analysts that chicory, dandelion and, probably, some other substances that are used for mixing with coffee, are readily deprived of coloux by a weak solution of chloride of lime (hypochlorite), and that this agent has very little action on coffee. When this method is adopted, a portion of the coffee should be gently boiled a short time in water, with a little carbonate of soda, so as to remove as much extractive as possible; after subsidence the liquor should be poured off, and the residuum washed with distilled water. When this has been done sufficiently, a weak solution of the hypochlorite is to be added, and allowed to remain, with occasional stirring, until decolouration has taken place, which will probably be in two or three hours. The coffee will then form a dark stratum at the bottom of the glass, and the chicory, a light, almost white stratum, floating above it, and showing a clear and sharp line of separation. The chicory after this operation is in a fine condition for microscopical examination, and both the upper and lower strata of the deposit can be examined for other substances. Although the lower stratum may be dark coloured, and have the appearance of coffee, other substances may be present, and should be looked for.

I have recently met with a substance that is entirely new to me as a coffee substitute that is not affected by this treatment. 


\section{NOTE ON BUTTERINE.}

By Arthur Angeld, F.I.C., F.C.S.

Manufacturess have now succeeded in mixing foreign fats with butter. A large quantity of a compound called "Creamy Butterine" has recently been placed upon the market. It is a palatable article, has all the appearance and odour of a butter, and is therefore very different from the "butterines" and "oleomargarines" hitherto produced. It yields 92 per cent. of insoluble fatty acids, and under the micro-polariscope shows well-defined stellate crystals, thereby proving that some part of the compound has been fused. I may here mention that genuine butters made from scalded cream are crystalline in structure. 